\title{
Optimization of Latency for Color Interpolation Processor
}

\author{
T.Nistchala \\ M.Tech, DECS Gudlavalleru Engineering College, Gudlavalleru Andhra Pradesh \\ M. Kamaraju \\ Ph.D Professor \& Head ECE Gudlavalleru Engineering College, Gudlavalleru Andhra Pradesh
}

\section{T.Venkata Lakshmi}

Associate Professor Gudlavalleru Engineering College Gudlavalleru, Andhra Pradesh

\begin{abstract}
Recently digital cameras are integrated into many consumer electronic products, such as digital camera, smart TV etc. The colors red(R), green $(\mathrm{G})$ and blue (B) are sampled as one color in each pixel and is important to reconstruct the images from Color Filter Array (CFA) to full RGB formats. Many efficient high-quality algorithms are present for reconstruction. Among them color interpolation processor based on edge direction weighing was more efficient one. Even though its performance was improved using the pipeline schedule and time-sharing techniques, it is necessary to use two division and three multiplication operations to obtain edge-direction which increases the hardware cost and chip area. Hence a filter-based compensation methodology that includes Laplacian and Spatial sharpening filters is used which improves the edge information and reduce the blurring effect. In addition to this, the hardware cost and the usage of dividers and multipliers was successfully reduced using adders along with hardware sharing reconfigurable design techniques.
\end{abstract}

Keywords: color filter array, spatial sharpening, laplacian, CSLA, edge detection, enhancing, and interpolator.

\section{Introduction}

Image processing is most widely used in many fields, such as medical imaging, scanning techniques, printing skills, license plate recognition, face recognition, and so on. Generally, images are often corrupted by impulse noise in the image acquisition and transmission techniques. The noise may seriously affect the performance of image processing techniques. Therefore, an efficient denoising technique became a very important issue in image processing [1].Digital cameras are developed by a Charge Coupled Device (CCD) or a CMOS image sensor that can capture images by colour filter array (CFA) technique. Fig 1 shows a colour filter arrays called Bayer CFA, in which two colours are not present in each pixel. Hence, it is important to reconstruct the images from CFA to Full RGB formats [3]. The existing VLSI Architecture will provide the segmented RGB pixel values in order to reconstruct the original image [2].

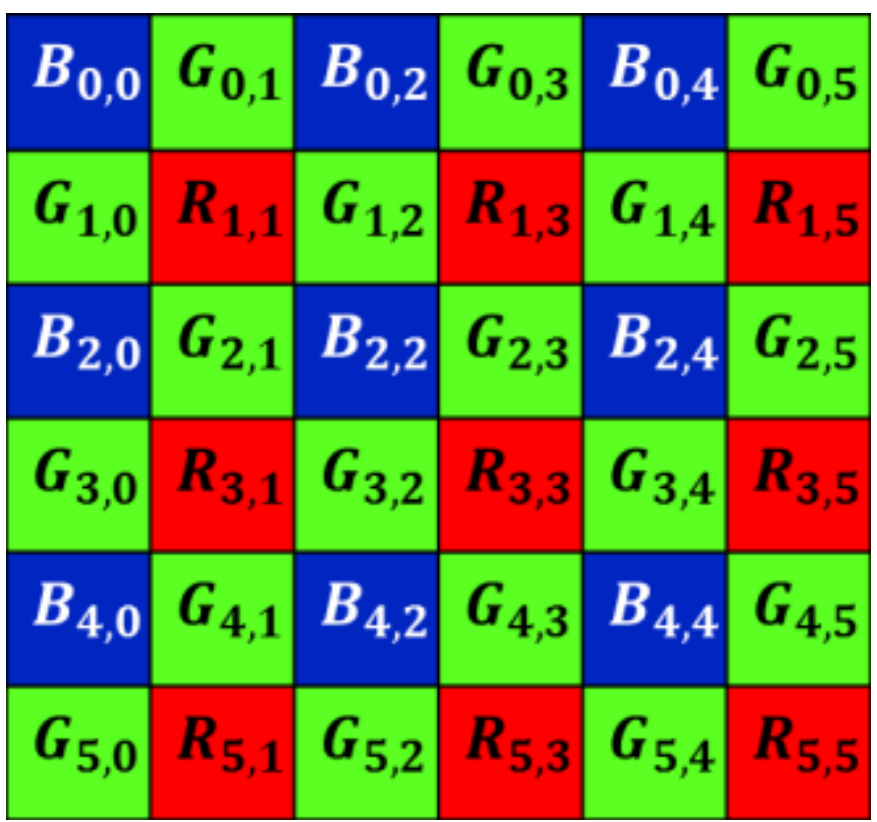

Fig: 1. Bayer color filter array (CFA).

\section{Literature Survey}

HSIA et al. [1] also presented a high-performance and lowcomplexity algorithms to develop a camera DSP system. Although this study achieved a better quality by using a white balance, a color space transformation, an auto gain control, an edge enhancement, and a color enhancement techniques, it is necessary to use the division and multiplication operations to find the gain and edge information. The hardware cost and chip area were efficiently increased due to the implementation of these dividers and multipliers. Low-cost and highperformance color demosaicking VLSI design was proposed by SHIAU et al. [2]. This design improved the quality of the interpolated images by using edge-information and interchannel correlations. By using pipeline architecture, the performance was greatly improved. Since the parameters of the edge-information and inter-channel correlations were divided by 2,4 , or 8 , it is unnecessary to use any divider to realize this design. The cost of the edge detector hardware is much less than the previous designs such as [1] and [2]. An edge estimation method using variance of the different colors 
was invented by CHUNG ET AL. [3]. In addition, an iterative K-SVD based algorithm [4] successfully improved the quality of interpolated images through iteration technique. Prior knowledge was used to improve the performance of demosaick images by MENON ET AL. [10]. The high-quality color interpolation algorithms, [1]-[10], mentioned above made great contributions in CFA images correction. However, these high-quality color interpolation algorithms are of high complexity and high memory requirement. Furthermore, these algorithms are not easy to be realized using VLSI technique. Several efficient techniques such as selecting [5], [6] or fusing [7] the information of the vertical and horizontal directions has also been presented. A gradient-base scheme with a Gaussian low-pass filter to improve the performance of the color interpolation was proposed by YUN ET AL. [8]. An effective scheme to enhance state-of-the art demosaicking methods using image spatial and spectral correlation was presented by CHANG ET AL. [9].

\section{Multiprocessing Technique with Carry Select Adder}

Fig 2 shows the block diagram of multiprocessing technique with carry select adder. It consists of a single color interpolation processor along with the carry select adder. There are three input images that are simultaneously given as input. The processor performs multiprocessing and hence it parallelly performs the edge enhancement operation on the three images and simultaneously presents them as the output [6].Hence the processor is highly intended to perform parallelism to reduce the delay considerably by using the carry select adder. Carry select adder is used to obtain a compromised reduction in delay and area.

Fig 3 shows the block diagram of the VLSI architecture for the color interpolation processor. It consists of seven main blocks: a register bank, an edge detector, a green color interpolator ( $\mathrm{G}$ interpolator), a red and blue colors interpolator model 1 (RB_M1 interpolator), red and blue colors interpolator model 2 (RB_M2 interpolator), a red and blue colors interpolator model 3 (RB_M3 interpolator), and a controller [11].

The register bank was designed to real-time provide fifteen pixels in CFA format for processing the $\mathrm{g}$ interpolator and three RB interpolators during each cycle [11].

It is designed with a two-line-buffer memory and constructed with fifteen shift registers. The proposed register bank is designed such that only one value of pixel from memory is received in each cycle time, and then provides fifteen values of CFA pixels as inputs for color interpolation. By adding this register bank, the proposed color interpolation processor achieves the memory access through pixel in and pixel out.

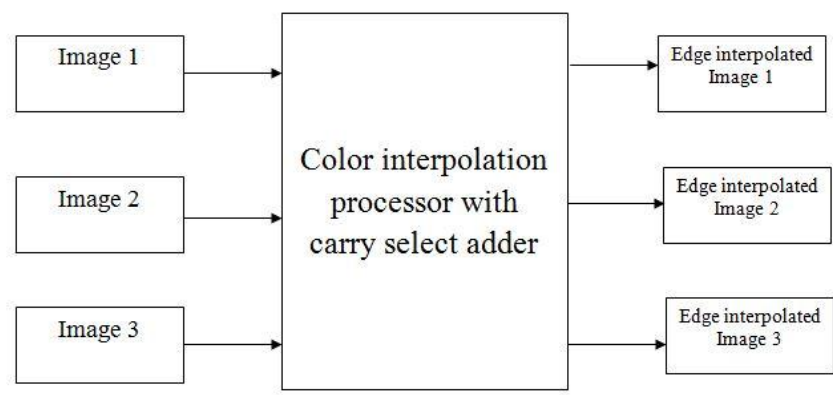

Fig: 2 Block diagram of multiprocessing technique with carry select adder

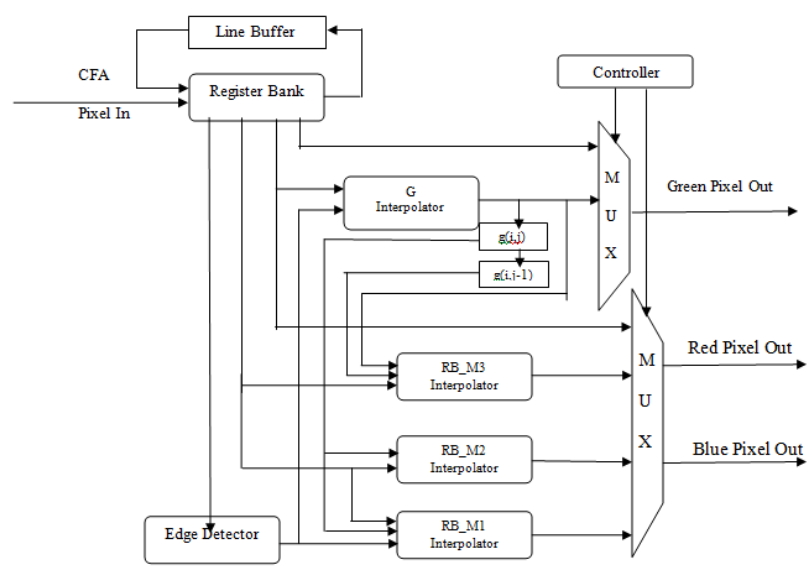

Fig: 3. VLSI architecture for the color interpolation processor.

\section{Register bank}

Fig 4 shows the architecture of register bank. The main purpose of this register bank is to store the input image pixel values that are arriving in a sequential manner at the input. The presence of line buffer will make sure that the pixel values are happened to be arriving at the serial order and it also provides a feedback path for the input pixels and assure the correct reading of the input image.

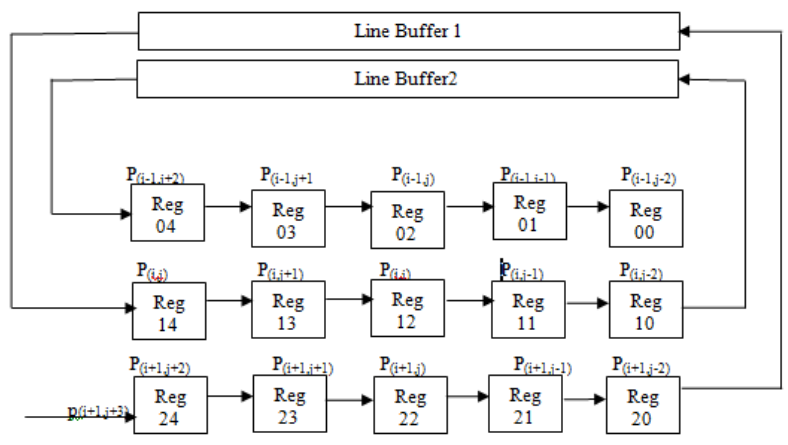

Fig 4: Architecture of the register bank 


\section{Edge detector}

Fig 5 shows the architecture of the edge detector. It consists of six absolute sub tractors (|sub|) and five adders (add).the eight input signals receive their inputs from the register bank [5]. After being processed by the edge detector, the values of TD, $\mathrm{DH}$, and DV at the position of $\mathrm{p}(\mathrm{i}, \mathrm{j})$ are produced for the green color interpolator and the first model of red and blue color interpolator (rb_m1). In addition, the output signals of the edge detector are also sent to the controller. The output signal of td provides information of the edge intensity. The other two output signals, DH and DV, give the direction information of the edges.

All adder blocks are designed using carry select adders. Carry select adder (CSLA) is one of the fastest adders that are used in many data-processing processor architectures to perform fast arithmetic functions. By using this CSLA, the area and power consumption is reduced in the design. This work uses a simple and efficient gate-level modification to significantly reduce the area and power.

The carry select adder divides the carry ripple adder into $\mathrm{m}$ parts, where each part consists of a duplicated $(\mathrm{n} / \mathrm{m})$-bit carry ripple adder pair, as shown in fig 7.

The carry ripple adder is constructed by cascading each single-bit full-adder. In this carry ripple adder, each full-adder starts its computation until the previous carry-out signal is ready. So, the critical path delay in a carry ripple adder is determined by its carry-out propagation path. For an n-bit fulladder as illustrated in fig. 6 the critical path is n-bit carry propagation path in the full-adders.

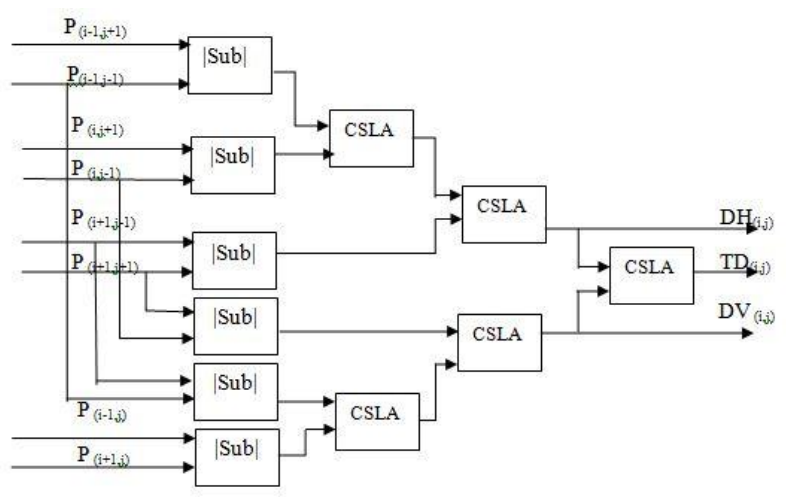

Fig: 5 Architecture of the edge detector

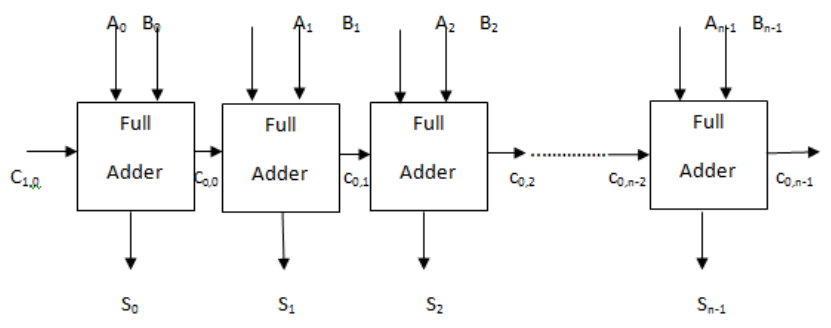

Fig: 6 N-bit full adder

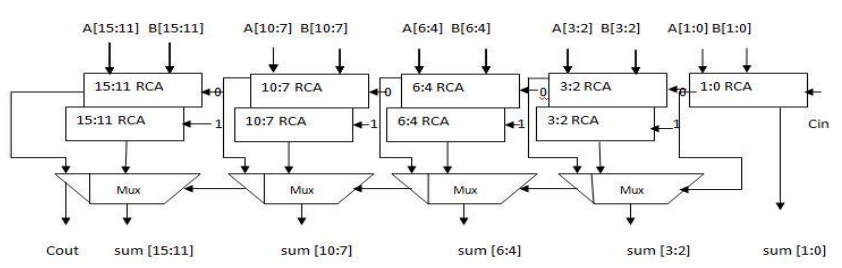

Fig: 7 16-bit carry select adder

\section{Green Color Interpolator}

Fig 8 shows the architecture of green colour interpolator. It consists of eight adders, one subtractor, four multiplexers, and five shifters. This design can be reconfigured as the function by multiplexer selection during each processing cycle. The control signals, which are sent to multiplexers, are produced by the controller according to the values of TD, DH, and DV. By using reconfigurable technique, the proposed green color interpolator has the characteristics of low cost, high flexibility and high performance. In order to shorten the critical path and improve the design performance, three registers were added in this architecture. The first register is used to store the result of the $g$ interpolator $g(i, j)$. It provides input signal $g(i, j)$ for the rb_m1 and rb_m2 modules. The second register is used to store the value of $g(i, j)$ that provides the input signal $g(i, j-1)$ for the rb_m3 module. Lastly, the third register is used to store the edge information of the TD, DH, and DV. By adding these three registers, this implementation is a pipeline architecture design. It achieves shortening the critical path and providing the interpolated green pixels of $g(i, j)$ and $g(i, j-1)$ for $r$ and $b$ interpolators to improve the quality of the interpolated $r(i, j)$ and $b(i, j)$ pixels.

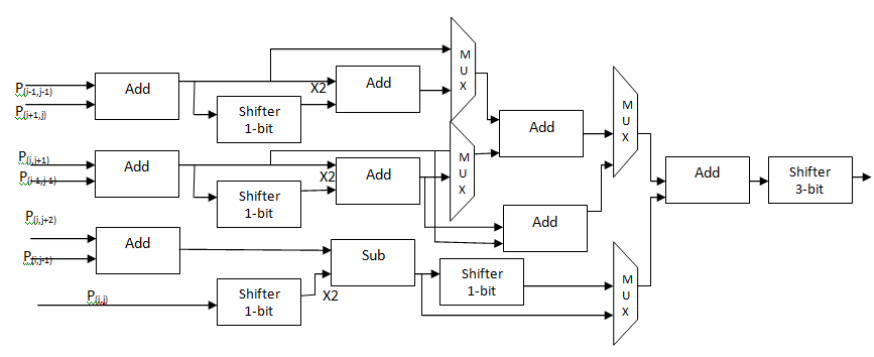

Fig 8 Architecture of green color interpolator

\section{Red and Blue color interpolators}

Fig 9 shows the architecture of the red and blue colour interpolator model1.It consists of nine serial adders, three 1bit shifters that performs left shift operation by 1-bit, one 2-bit shifter that performs 2-bits shifted left and 3-bit shifter that is being used to perform left shift. There are two multiplexers in the design that takes two inputs and provides single output for the shifter and one subtractor.

Fig 10 shows the architecture of the red and blue colour interpolator model2.It consists of adders, shifters and subtractor.

Fig 11 shows the RB_M3 interpolator architecture. RB_M3 interpolator performs like RB_M2. It consists of three adders, 
one subtractor, and one shifter and has the lowest cost module among the four interpolators.

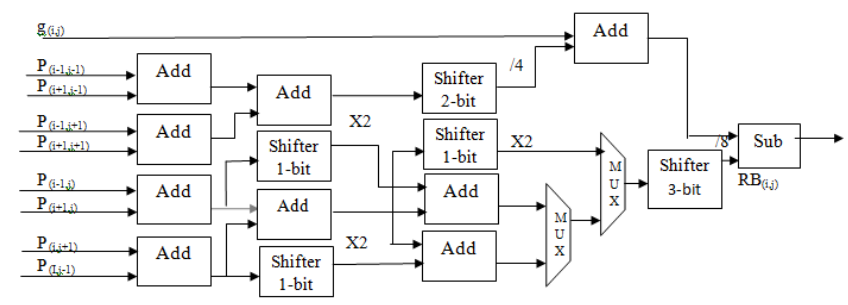

Fig. 9 Architecture of the red and blue color interpolator model 1 (RB_M1 interpolator)

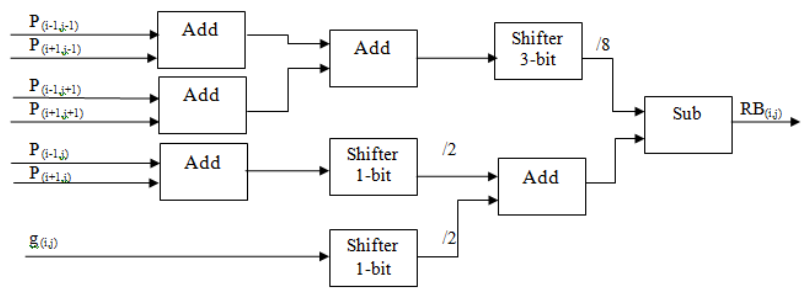

Fig. 10 Architecture of the red and blue color interpolator model 2 (RB_M2 interpolator)

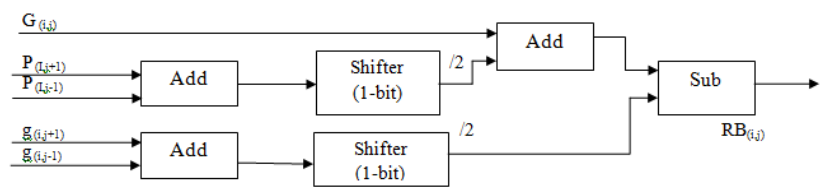

Fig. 11 Architecture of the red and blue colours interpolator model 3 (RB_M3 interpolator)

\section{Controller}

The controller provides control signals to the multiplexer for selecting input data for the interpolators and is capable of sending reconfigurable control signals for changing the architecture of the interpolators. Moreover, the controller must monitor its input and output data access with the memory to fit the performance of pixel-in and pixel-out. Finally, the proposed colour interpolation processor achieves high performance and high throughput

\section{Implementation}

This paper is implemented using the MATLAB dip tool and MODELSIM QUARTUS simulation tool. The input image pixel values are read from the MATLAB dip tool. The respective pixel values are stored in a buffer and are given as digital inputs to the MODELSIM tool in order to process them according to the architecture of the multiprocessing color interpolation processor. More than one image can be processed using this architecture which provides parallelism [11] with less delay when compared to the processing of single image at a time. Thus these architectures are developed and applied to the pixel values, which are read from MATLAB, dip tool and are processed using MODELSIM software.

\section{Flow chart}

Fig 12 shows the step by step implementation of the edge detection and enhancement using multiprocessing technique with carry select adder. Initially the input image is read using the MATLAB software. The read pixel values are converted into binary values and are stored in the form of a text document which is provided as inputs to the MODELSIM tool directly from the MATLAB by establishing a connection between them. When the values are present in the text file then the MODELSIM starts simulation of the pixel values. Once the simulation is completed the resultant pixel values are again stored in another text document and the connection that is being established is interrupted to display the output image in the MATLAB.

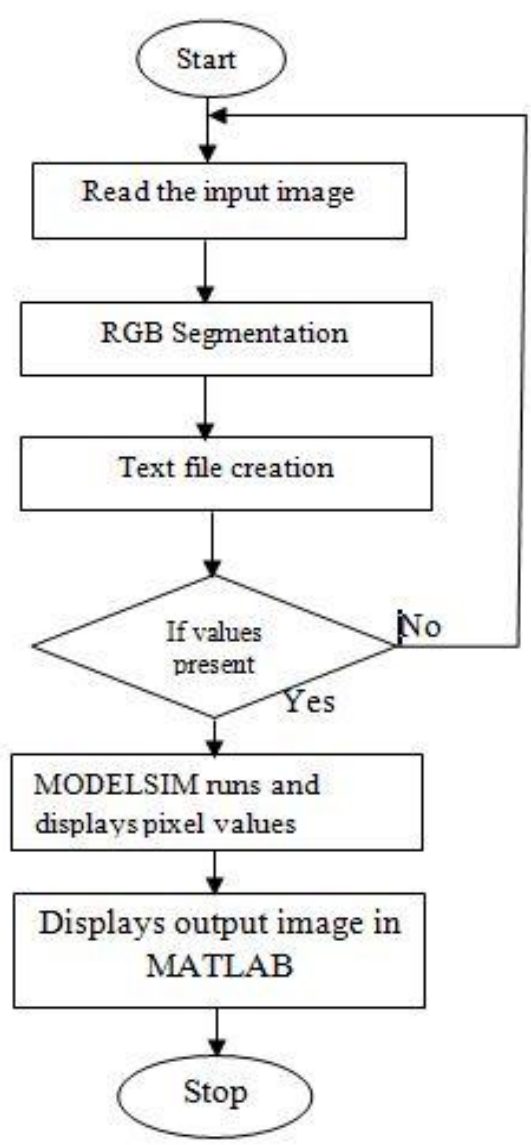

Fig: 12 Flow chart

\section{Results}

Fig 13 shows the simulation result of the input pixel values for the multiprocessing technique with carry select. The pixel values are processed using a test bench. All the intermediate signals along with the clock generations and the final binary values that are stored in the text document are displayed in the simulation. 
Fig 14 shows the input images that are being edge enhanced using the multiprocessing color interpolation processor. As shown in the figure the images that are produced as input to the processor are enhanced at its edges and are enhanced to have a clear distinction between the original image and its background. The multiprocessor mainly performs parallelism and processes all the given input images at a time simultaneously.

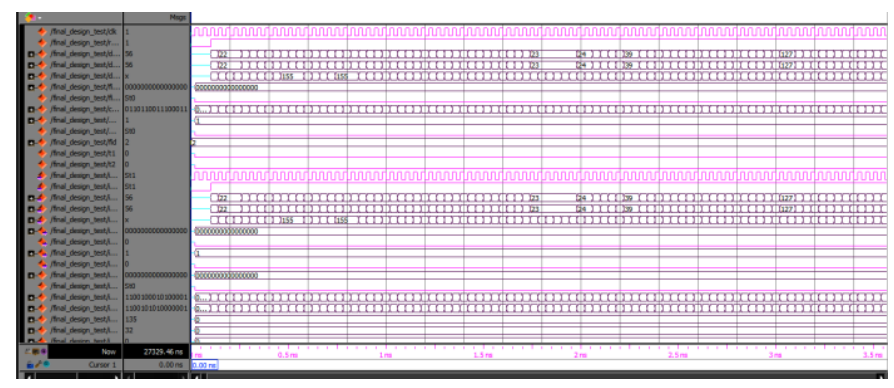

Fig: 13 Simulation result of the color interpolation processor with multiprocessing technique

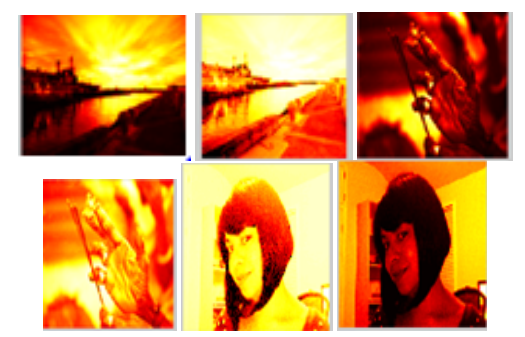

Fig: 14 Input and output edge interpolated color images

\section{Comparison}

The below Table 1 shows the comparison results of processing times using the existing processor without carry select adder and the multiprocessing with carry select adder techniques. From the table it is clear that the delay is reduced more for three images provided as input and obtaining simultaneously at the output. Hence the carry select adder technique will produce less delay for multiple images which improves the speed of operation of the design.

Table: 1 latency comparison in MATLAB

\begin{tabular}{|l|l|lr|}
\hline & $\begin{array}{l}\text { Latency using } \\
\text { processor without carry } \\
\text { select adder Technique }\end{array}$ & $\begin{array}{l}\text { using } \\
\text { multiprocessing with carry } \\
\text { select adder architecture } \\
\text { Technique }\end{array}$ \\
\hline $\begin{array}{l}\text { Single } \\
\text { image }\end{array}$ & 64.58 seconds & 43.47 seconds \\
\hline $\begin{array}{l}\text { Three } \\
\text { images }\end{array}$ & 91.87 seconds & 62.31 seconds \\
\hline
\end{tabular}

\section{Conclusion}

Finally, this work adaptive edge interpolated image scalar is developed, which offers low complexity, low memory requirement and high speed. In this work extra pipelines are introduced at the end of gradient based carry select addition for improving speed. These parallel processing offers reduced critical path, hence improved the speed of circuit operation and thus providing low latency for multiprocessing operations..The latency is reduced by $30 \%$ using the multiprocessing carry select adder technique when compared to that of the existing processor architecture technique.

\section{References}

1. S. C. Hsia, and P. S. Tsai, "VLSI implementation of camera digital signal processor for document projection system," in Proc. IEEE Int. Conf. Signal Processing System (ICSPS), Jul. 2010, pp. 657-660.

2. Y. H. Shiau, P. Y. Chen, and C. W. Chang, "An areaefficient color demosaicking scheme for VLSI architecture," International Journal of Innovative Computing, Information and Control, Vol.7, No.4, pp.1739-1752, Apr. 2011.

3. K. Hirakawa and T. W. Parks, "Adaptive homogeneity-directed demosaicking algorithm," IEEE Trans. Image Process.,vol. 14, no. 3, pp. 360369, Mar. 2005.

4. D. Menon, S. Andriani, and G. Calvagno, "Demosaicing with directional filtering and a posteriori decision," IEEE Trans. Image Process., vol. 16, no. 1, pp. 132-141, Jan. 2007.

5. L. Zhang and $\mathrm{X}$. $\mathrm{Wu}$, "Color demosaicking via directional linear minimum mean square-error estimation," IEEE Trans. Image Process., vol. 14, no. 12, pp. 2167-2177, Dec. 2005.

6. S. H. Yun, J. H. Kim, and S. Kim, "Color interpolation by expanding a gradient method," IEEE Trans. Consumer Electronics, vol. 54, no. 4, pp. 1531-1539, Nov. 2008.

7. L. Chang, and Y. P. Tan, "Effective use of spatial and spectral correlations for color filter array demosaicking," IEEE Trans. Consumer Electronics, vol. 50, no. 1, pp. 355-365, Feb. 2004.

8. K.-H. Chung and Y.-H. Chan, "Color demosaicing using variance of color differences," IEEE Trans. Image Process., vol. 15, no. 10, pp. 2944-2955, Oct. 2006.

9. J. Mairal, M. Elad, and G. Sapiro, "Sparse representation for color image restoration," IEEE Trans. Image Process., vol. 17, no. 1, pp. 53-69, Jan. 2008.

10. D. Menon and G. Calvagno, "Regularization Approaches to Demosaicking," IEEE Trans. Image Process. vol. 18, no. 10, pp. 2209-2220, Oct. 2009.

11. Shin-Lun Chen and En-Di Ma, "VLSI Implementation of an adaptive edge enhanced color interpolation processor for real time video application", IEEE Transactions on circuits and systems for video technology vol no. 10.11/TCSVT.2014.2317890 